\title{
The neuropeptide genes SST, TAC1, HCRT, $N P Y$, and GAL are powerful epigenetic biomarkers in head and neck cancer: a site-specific analysis
}

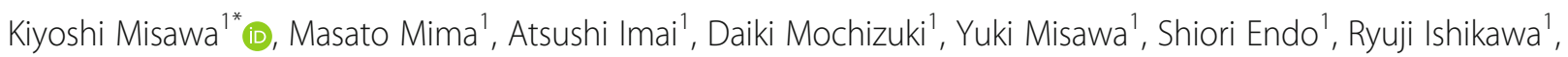
Takeharu Kanazawa ${ }^{2}$ and Hiroyuki Mineta ${ }^{1}$

\begin{abstract}
Background: Staging and pathological grading systems are convenient but imperfect predictors of recurrence in head and neck squamous cell carcinoma (HNSCC). Identifying biomarkers for HNSCC that will progress and cause death is a critical research area, particularly if the biomarker can be linked to selection of patients. Therefore, to identify potential alternative prognostic markers, we investigated the methylation status of five neuropeptide gene promoters. The promoter methylation status was determined by quantitative methylation-specific PCR in 230 cases of HNSCC; 58 hypopharynx, 45 larynx, 56 oropharynx, and 71 oral cavity tumor samples were studied.
\end{abstract}

Results: The somatostatin (SST), tachykinin precursor 1 (TAC1), hypocretin neuropeptide precursor (HCRT), neuropeptide $Y$ (NPY), and galanin (GAL) promoters were methylated in $84.3,63.5,32.6,28.3$, and $20.0 \%$, respectively, of the samples. The mean number of methylated genes per sample was 2.29 (range, $0-5$ ). Disease-free survival was lower in patients with 3-5 methylated genes than in those with $0-2$ methylated genes (log-rank test, $P=0.007$ ). In multivariate Cox proportional hazards analysis, TAC1 and GAL promoter methylation independently predicted recurrence (odds ratios 1.620, 95\% confidence interval $[\mathrm{Cl}] 1.018-2.578, P=0.042$, and odds ratios $1.692,95 \% \mathrm{Cl} 1.063-2.694, P=0.027$, respectively). In patients with oral cancer, TAC1 methylation showed the best correlation with poor survival (odds ratio 4.427, $95 \% \mathrm{Cl} 1.634-12.00, P=0.003)$. Similar findings were observed for HCRT and GAL in patients with laryngeal cancer and oropharyngeal cancer, respectively.

Conclusion: In this study, we demonstrated the methylation status of the neuropeptide-encoding genes SST, TAC1, HCRT, NPY, and GAL and its relationship with recurrence and survival in HNSCC. These methylation changes may serve as potential molecular markers for defining the risk and prognosis of HNSCC.

Keywords: SST, TAC1, HCRT, NPY, GAL, Head and neck cancer, Epigenetic markers, Site-specific analysis

\section{Background}

Neuropeptides and their receptors are important messenger molecules that carry information between neurons; they can act as neurohormones, neurotransmitters, and neuromodulators and maintain physiological homeostasis $[1,2]$. Neuroendocrine peptides play essential roles in the regulation of gastrointestinal endocrine

\footnotetext{
* Correspondence: kiyoshim@hama-med.ac.jp

${ }^{1}$ Department of Otolaryngology/Head and Neck Surgery, Hamamatsu

University School of Medicine, 1-20-1 Handayama, Hamamatsu, Shizuoka

431-3192, Japan

Full list of author information is available at the end of the article
}

and exocrine secretion, motility, and mucosal immunity. Moreover, some neuroendocrine peptides such as gastrin, vasoactive intestinal peptide, and neurotensin have been implicated in the modulation of human tumorigenesis [3, 4]. Most neuropeptides exert their effect through G protein-coupled receptors (GPCRs), with some exceptions [4]. GPCRs belong to a superfamily of cell surface signaling proteins that play a pivotal role in many physiological functions and multiple diseases [5]. Recent data have indicated that many GPCRs and their ligands are involved in cancer initiation and progression, 
including processes such as aberrant cell proliferation, invasion, metastasis, migration, adhesion, and angiogenesis [6]. The expression and secretion of neuropeptides by tumors has attracted increasing interest, as these peptides have been found to influence tumor proliferation and to be correlated with the appearance of characteristic clinical symptoms [7].

Staging and pathological grading systems are convenient but imperfect predictors of recurrence in head and neck squamous cell carcinoma (HNSCC). In HNSCC, methylation of gene promoters is a common mechanism of transcriptional silencing [8]. We recently defined the methylation profiles of the neuropeptide genes somatostatin (SST), tachykinin precursor 1 (TAC1), and galanin $(G A L)$ and their cognate receptor gene members in HNSCC tumors
[9-11]; however, further studies were required because of the small sample size and the lack of discrimination between the sites of origin of primary tumors. Nevertheless, it is evident that the neuropeptide system functions as a major mechanism in HNSCC carcinogenesis.

The current findings provide novel direct epigenetic evidence for the involvement of SST, TAC1, hypocretin neuropeptide precursor $(H C R T)$, neuropeptide $\mathrm{Y}$ $(N P Y)$, and GAL in the process of tumor suppression in humans. The association between the methylation status of the five genes and clinicopathological characteristics (e.g., tumor location and lymph node metastasis) was also assessed. To our knowledge, this study is the first to implicate neuropeptide gene methylation in the genesis of HNSCC.

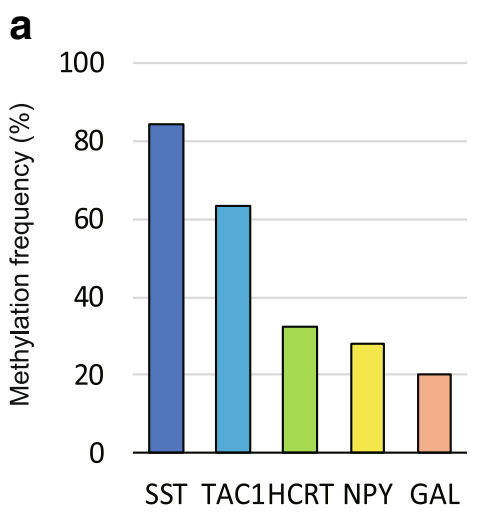

C

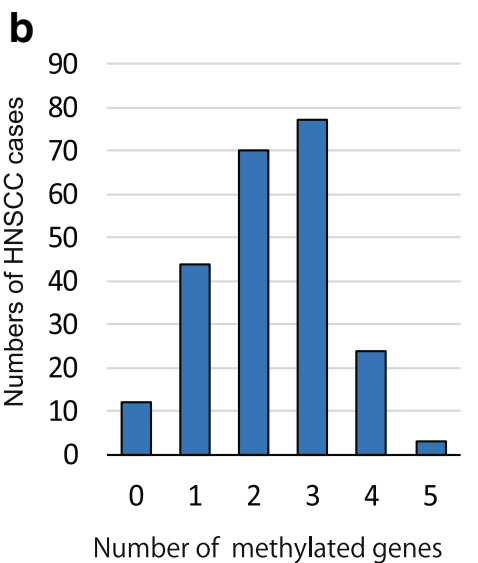

Number of methylated genes

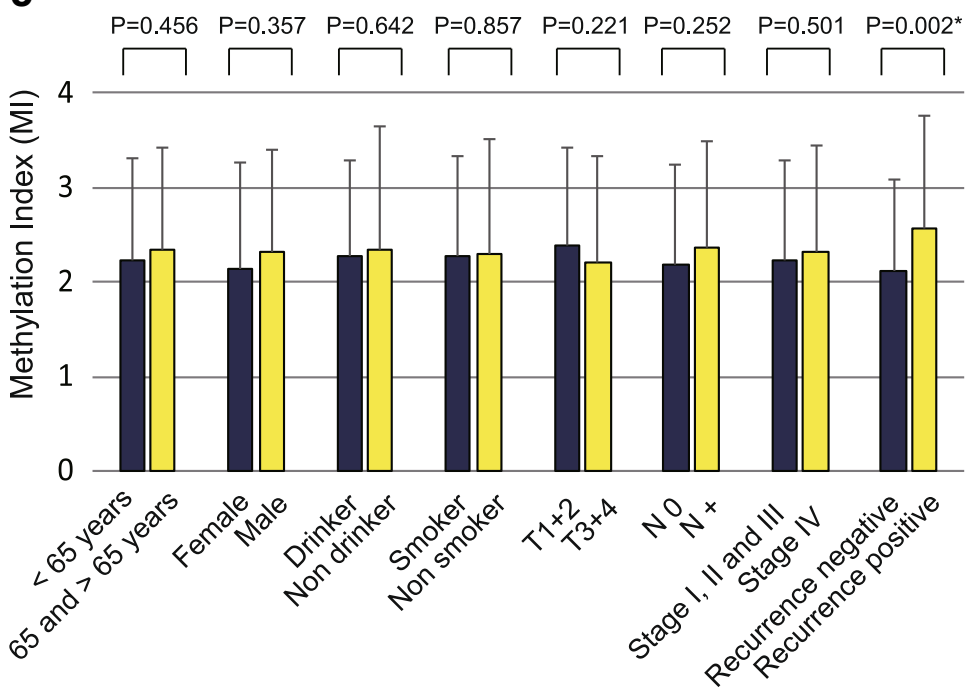

Fig. 1 Methylation of the SST, TAC1, HCRT, NPY, and GAL gene promoters in 230 HNSCC samples. a Bar graph showing the methylation frequencies of the five genes. $\mathbf{b}$ Bar graph comparing the number of HNSCC cases to the number of methylated genes. $\mathbf{c}$ Double bar graph showing the methylation indices (Mls) according to the selected clinical parameters. The mean Ml for each parameter was determined by using Student's $t$ test. Mean and standard deviation are also indicated, and statistical comparisons between groups are depicted. A probability of $<0.05\left({ }^{*} P<0.05\right)$ was considered to represent a statistically significant difference 


\section{Methods}

\section{Tumor samples}

All clinical specimens were surgically obtained from primary HNSCCs $(n=230)$ at Hamamatsu University School of Medicine. The samples were obtained soon after diagnosis and were thus from untreated tumors. All patients provided written informed consent, and the study protocol was approved by the Institutional Review Board of the Hamamatsu University School of Medicine. Pertinent information including age, sex, smoking status, alcohol consumption, tumor site, size, lymph node status, and clinical stage was obtained from the patients' medical records. The male to female ratio in the patient cohort was 193:37. The mean age was 65.5 years (range, $32-93$ years). Primary tumors were in the hypopharynx $(n=58)$, larynx $(n=45)$, oropharynx $(n=56)$, or oral cavity $(n=71)$.

\section{Quantitative methylation-specific PCR (Q-MSP) analysis}

Extraction and bisulfite conversion of genomic DNA from 230 primary HNSCC and 36 noncancerous mucosal samples were performed using the MethylEasy Xceed Rapid DNA Bisulfite Modification Kit (TaKaRa, Tokyo, Japan) per the manufacturer's instructions [12]. The methylation levels of the $\mathrm{CpG}$ islands in the promoters of the SST, TAC1, HCRT, $N P Y$, and GAL genes were determined via Q-MSP with the TaKaRa Thermal Cycler Dice Real Time System TP800 (TaKaRa); the primer sets are listed in Additional file 1: Table S1. Exon structure and CpG sites within expanded views of the promoter region relative to the transcription start site (TSS) are presented in Additional file 2: Figure S1. A standard curve was constructed by plotting known concentrations of serially diluted EpiScope Methylated HeLa gDNA (TaKaRa). The normalized methylation value (NMV) was determined as follows: $\mathrm{NMV}=$ (Target gene-S/Target gene-FM)/(ACTB-S/ACTB-FM), where Target gene-S and Target gene-FM represent the target gene methylation levels in the tumor sample and universal methylated DNA control, respectively, and

Table 1 Distribution of methylation status by selected epidemiologic and clinical characteristics

\begin{tabular}{|c|c|c|c|c|c|c|c|c|c|c|c|c|c|c|c|c|c|}
\hline \multicolumn{5}{|c|}{ Characteristics } & \multicolumn{4}{|l|}{ Age } & \multicolumn{5}{|c|}{ Gender } & \multicolumn{4}{|c|}{ Smoking status } \\
\hline Gene & \multicolumn{2}{|c|}{ Methylation status } & \multicolumn{2}{|c|}{ Overall(\%) } & $<65$ & \multicolumn{2}{|l|}{$>65$} & $P^{\dagger}$ & \multicolumn{2}{|c|}{ Female } & Male & \multicolumn{2}{|l|}{$P^{\dagger}$} & Smoker & \multicolumn{2}{|c|}{ Non-smoker } & $P^{\dagger}$ \\
\hline \multirow[t]{2}{*}{ SST } & \multicolumn{2}{|l|}{ Yes } & \multicolumn{2}{|c|}{ 194(84.3) } & 81 & \multicolumn{2}{|l|}{113} & & \multicolumn{2}{|l|}{29} & 165 & & & 146 & \multicolumn{2}{|l|}{48} & \\
\hline & \multicolumn{2}{|l|}{ No } & \multicolumn{2}{|c|}{$36(15.7)$} & 17 & \multicolumn{2}{|l|}{19} & 1 & \multicolumn{2}{|l|}{8} & 28 & \multicolumn{2}{|l|}{1} & 26 & \multicolumn{2}{|l|}{10} & 1 \\
\hline \multirow[t]{2}{*}{ TAC1 } & \multicolumn{2}{|l|}{ Yes } & \multicolumn{2}{|c|}{$146(63.5)$} & 59 & \multicolumn{2}{|l|}{87} & & \multicolumn{2}{|l|}{18} & 128 & & & 118 & \multicolumn{2}{|l|}{28} & \\
\hline & No & & $84(36.5)$ & & 39 & 45 & & 1 & 19 & & 65 & 0.061 & & 54 & 30 & & $0.007^{*}$ \\
\hline HCRT & Yes & & $75(32.6)$ & & 35 & 40 & & & 7 & & 68 & & & 52 & 23 & & \\
\hline & No & & $155(67.4$ & & 63 & 92 & & 1 & 30 & & 125 & 0.057 & & 120 & 35 & & 1 \\
\hline NPY & Yes & & $65(28.3)$ & & 24 & 41 & & & 11 & & 54 & & & 44 & 21 & & \\
\hline & No & & $165(71.7$ & & 74 & 91 & & 0.302 & 26 & & 139 & 1 & & 128 & 37 & & 1 \\
\hline GAL & Yes & & $46(20.0)$ & & 19 & 27 & & & 14 & & 32 & & & 30 & 16 & & \\
\hline & No & & $184(80.0$ & & 79 & 105 & & 0.869 & 23 & & 161 & 1 & & 143 & 42 & & 1 \\
\hline Charac & teristics & & Alcohol & exposure & & Tumo & r size & & $\begin{array}{l}\text { Lym } \\
\text { statc }\end{array}$ & $\begin{array}{l}\text { iph no } \\
\text { us }\end{array}$ & de & Stage & & & Recurren & ce events & \\
\hline Gene & $\begin{array}{l}\text { Methylation } \\
\text { status }\end{array}$ & Overall(\%) & Drinker & Non-drinker & $P^{\dagger}$ & T1-2 & T3-4 & $P^{\dagger}$ & No & $\mathrm{N}+$ & $P^{\dagger}$ & I, II, III & IV & $P^{\dagger}$ & Positive & Negative & $P^{\dagger}$ \\
\hline SST & Yes & 194(84.3) & 140 & 54 & & 95 & 99 & & 80 & 114 & & 86 & 108 & & 78 & 116 & \\
\hline & No & $36(15.7)$ & 25 & 11 & 1 & 16 & 20 & 0.717 & 19 & 17 & 0.206 & 17 & 19 & 1 & 13 & 23 & 0.713 \\
\hline TAC1 & Yes & $146(63.5)$ & 112 & 34 & & 70 & 76 & & 59 & 87 & & 64 & 82 & & 65 & 81 & \\
\hline & No & $84(36.5)$ & 53 & 31 & 1 & 41 & 43 & 1 & 40 & 44 & 1 & 39 & 45 & 1 & 26 & 58 & 0.051 \\
\hline HCRT & Yes & $75(32.6)$ & 55 & 20 & & 39 & 36 & & 28 & 47 & & 30 & 45 & & 37 & 38 & \\
\hline & No & $155(67.4)$ & 110 & 45 & 0.756 & 72 & 83 & 0.482 & 71 & 84 & 0.257 & 73 & 82 & 0.326 & 54 & 101 & 1 \\
\hline NPY & Yes & $65(28.3)$ & 44 & 21 & & 37 & 28 & & 32 & 33 & & 30 & 35 & & 26 & 39 & \\
\hline & No & $165(71.7)$ & 121 & 44 & 1 & 74 & 91 & 0.109 & 67 & 98 & 1 & 73 & 92 & 1 & 65 & 100 & 1 \\
\hline GAL & Yes & $46(20.0)$ & 25 & 21 & & 23 & 23 & & 18 & 28 & & 20 & 26 & & 27 & 19 & \\
\hline & No & 184(80.0) & 140 & 44 & 1 & 88 & 96 & 1 & 81 & 103 & 0.619 & 83 & 101 & 0.87 & 64 & 120 & $0.004^{*}$ \\
\hline
\end{tabular}


ACTB-S and ACTB-FM represent the ACTB (which encodes $\beta$-actin) methylation levels in the sample and control, respectively. Analysis was performed using the software (version 1.03A) for the Thermal Cycler Dice Real Time System TP800 (TaKaRa), according to the manufacturer's directions [13].

\section{Collection of publicly available data from The Cancer Genome Atlas (TCGA)}

Aberrant DNA methylation data available in the TCGA (November 2017) were collected via the MethHC database (http://methhc.mbc.nctu.edu.tw/php/index.php) by using the Infinium HumanMethylation450 platform (Illumina, Inc., San Diego, CA, USA) and were expressed as $\beta$ values. The $\beta$ value is a number between 0 (not methylated) and 1 (completely methylated) that represents the ratio of methylated allele intensity and overall intensity [14].

\section{Data analysis and statistics}

The Q-MSP results and patient characteristics (age of onset, sex, alcohol consumption, smoking status, tumor size, tumor stage, clinical stage, lymph node status, and recurrence) were compared by using the chi-squared test and Student's $t$ test. Receiver operating characteristic (ROC) curve analysis was performed using the NMVs for $36 \mathrm{HNSCC}$ and 36 adjacent normal mucosal samples in the Stata/SE 13.0 system (Stata Corporation, TX, USA). The area under the ROC curve indicated the optimal sensitivity and specificity cutoff levels for distinguishing between the methylation levels in normal and HNSCC tissue, and the NMV thresholds were calculated for each target gene (Additional file 3: Figure S2). The cutoff values were used to determine the methylation frequencies of the target genes. The overall methylation rates in the individual samples were determined by calculating the methylation index (MI). MI was defined as the ratio of the number of methylated genes to the number of tested genes in each sample [13].

Disease-free survival (DFS) was measured from the date of the initial treatment to the date of diagnosis of locoregional recurrence or distant metastasis. The Kaplan-Meier test was used to calculate survival probability, and the log-rank test was used to compare survival rates. The prognostic value of methylation status was assessed by performing multivariate Cox proportional hazards analysis which was performed with adjustment for age ( $\geq 65$ versus $<65$ years), sex, smoking status, alcohol intake, and tumor stage (I and II versus III and IV). Differences with $P<0.05$ were considered significant. All statistical analyses were performed using StatMate IV software (ATMS Co. Ltd., Tokyo, Japan).

\section{Results}

Methylation status of neuropeptide gene promoters

Q-MSP was used to assess the promoter methylation status of five genes encoding neuropeptide receptors in 230 primary HNSCC samples. The methylation rates for the five genes were as follows: SST, 84.3\%; TAC1, 63.5\%;

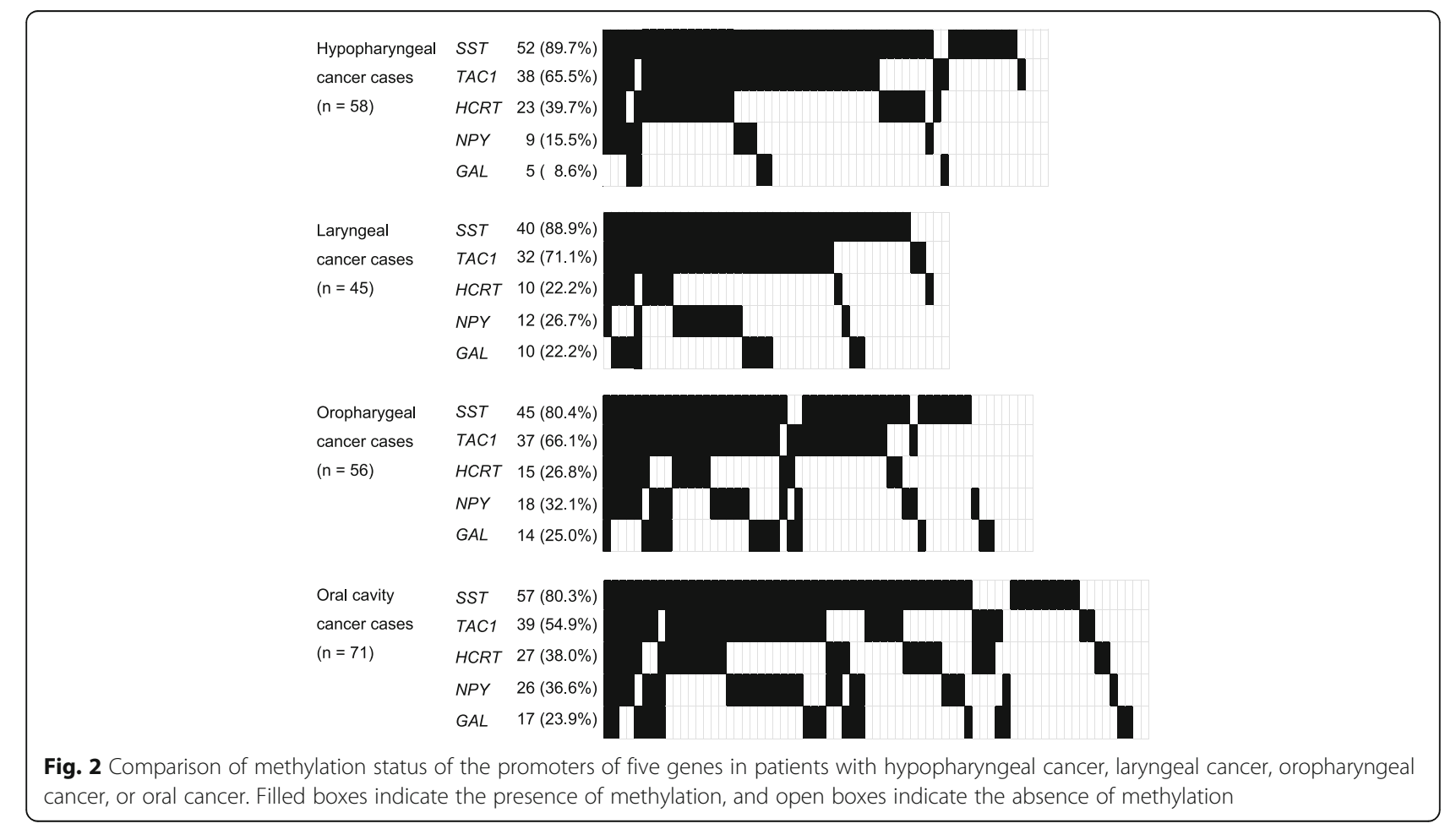


HCRT, 32.6\%; NPY, 28.3\%; and GAL, 20.0\% (Fig. 1a). At least one of these genes was methylated in most of the samples (218 of 230 samples, 94.8\%). The mean number of methylated genes per sample was 2.29 (range, 0-5; Fig. 1b).

\section{Correlation between the methylation status of neuropeptide} gene promoters and clinicopathological parameters

The associations between the methylation status of the target genes and the clinicopathological features of the patients are summarized in Table 1 . The methylation of the GAL promoter was significantly correlated with recurrence events $(P=0.004)$. A trend toward higher recurrence rate was observed for patients with methylated TAC1 $(P=0.051$; Table 1). Methylation of TAC1 was associated with smoking status $(P=0.007)$.

The mean differences in the MI according to the age of onset, sex, alcohol consumption, smoking habit, tumor size, lymph node status, clinical stage, and recurrence are illustrated in Fig. 1c. The MI was significantly higher in recurrence-positive cases than in recurrencenegative cases $(P=0.002$; Fig. $1 \mathrm{c})$.

\section{Site-specific analysis of the methylation status of neuropeptide gene promoters}

Site-specific methylation frequencies of five genes for the hypopharynx $(n=58)$, larynx $(n=45)$, oropharynx $(n=56)$, and oral cavity $(n=71)$ are shown in Fig. 2 . There was no significant association between clinicopathological characteristics and the MI in patients with hypopharyngeal cancer (Fig. 3a). Among laryngeal cancers, the MI was significantly higher in T1-2 than in T3-4 $(P=0.018$; Fig. $3 b)$. In patients with oropharyngeal cancer, no correlation was found between the MI and clinicopathological characteristics (Fig. 3c). We found that the MI was significantly higher in recurrence-positive cases than in recurrence-negative cases of oral cavity cancers $(P=0.004$; Fig. $3 \mathrm{~d})$.

\section{Kaplan-Meier analysis}

The Kaplan-Meier survival curves for each of the five target genes are shown in Fig. 4. DFS time did not
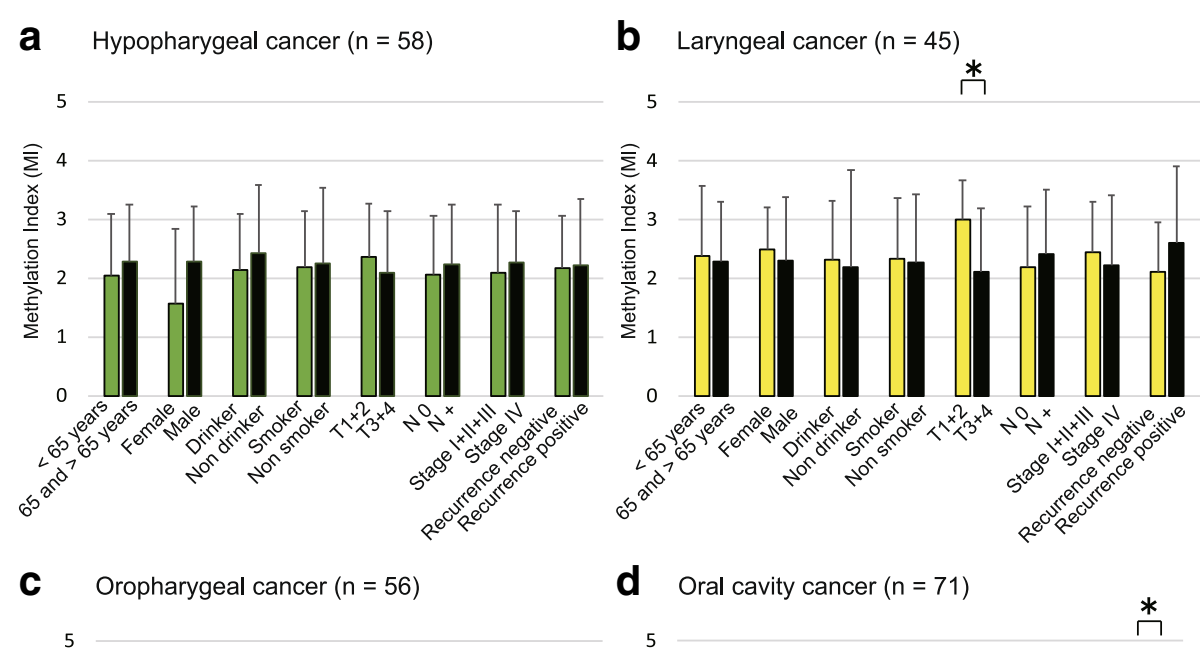

C Oropharygeal cancer $(n=56)$
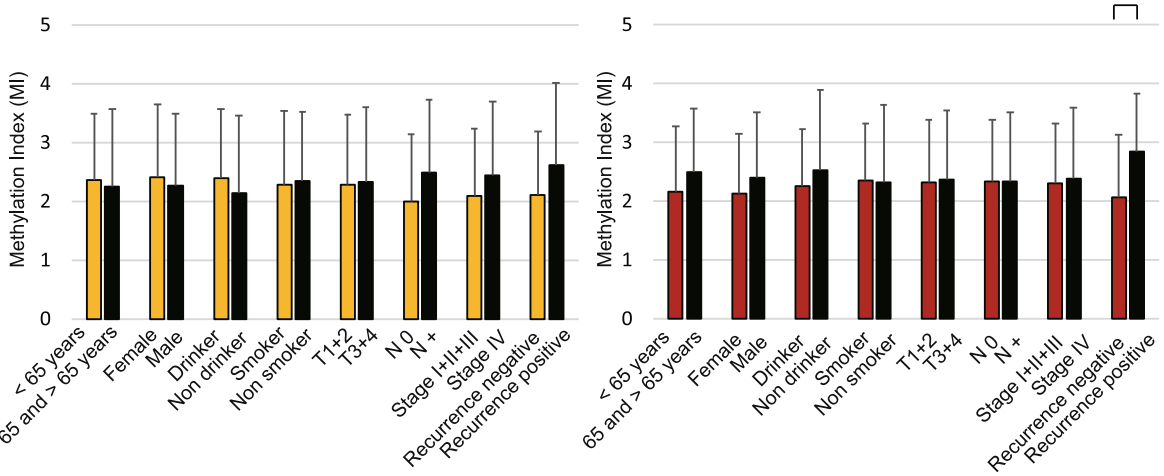

Fig. 3 Association between Ml and the selected clinical parameters. The mean Ml for the various groups was compared using Student's $t$ test. Association between $\mathrm{Ml}$ and selected epidemiologic and clinical characteristics: a hypopharyngeal cancer: no differences were noted with regard to any of the clinical characteristics; $\mathbf{b}$ laryngeal cancer: statistically significant differences were found for the associations between Ml and tumor size; c oropharyngeal cancer: no differences were noted with regard to any of the clinical characteristics; and $\mathbf{d}$ oral cavity cancer: statistically significant differences were found for the associations between $\mathrm{Ml}$ and recurrence events (positive versus negative). Mean and standard deviation are also indicated, and statistical comparisons between groups are depicted. A probability of $<0.05\left({ }^{*} P<0.05\right)$ was considered to represent a statistically significant difference 


\section{a}

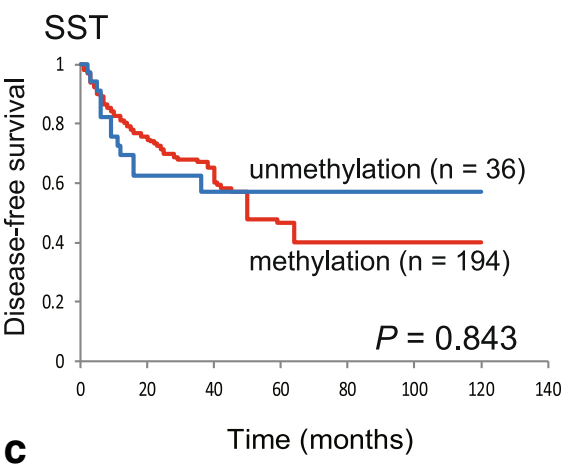

HCRT

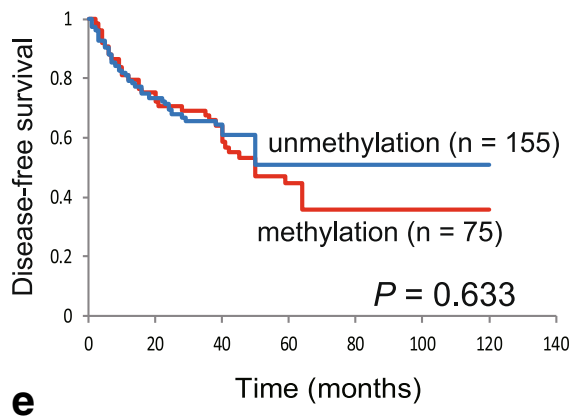

e

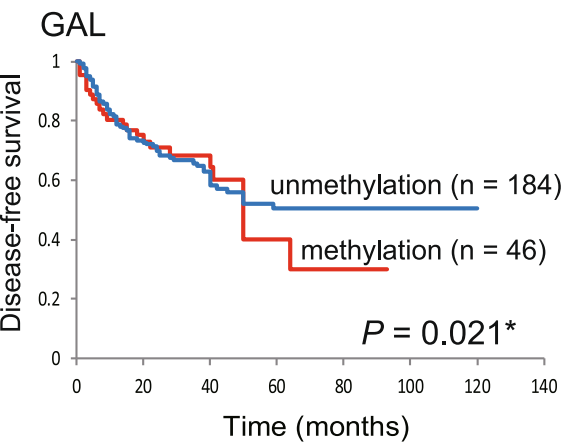

b
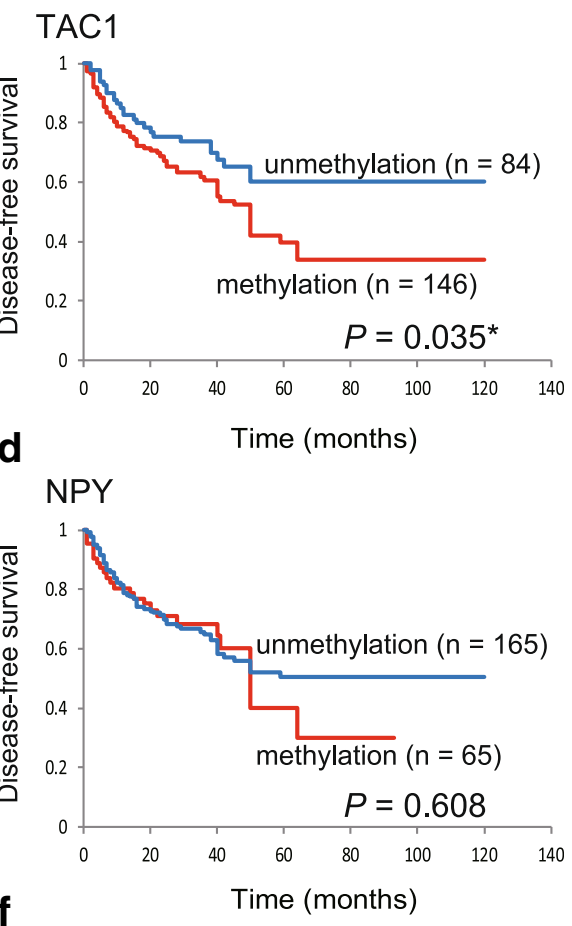

Full janel

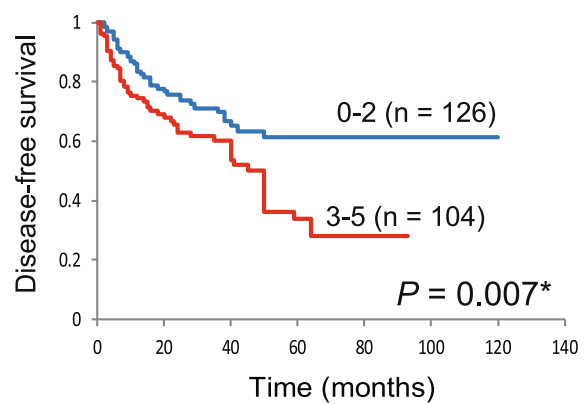

Fig. 4 Kaplan-Meier survival curves for the 230 patients with HNSCC, according to the methylation status of the five target genes. Disease-free survival for a SST, b TAC1, c HCRT, d NPY, and e GAL in the case of methylated (red lines) and unmethylated (blue lines) genes. $\mathbf{f}$ Joint analysis of the five genes. Blue line: patients with 0-2 methylated genes; red line: patients with 3-5 methylated genes. A probability of $<0.05$ ( ${ }^{*} P<0.05$ ) was considered to represent a statistically significant difference

significantly differ between patients with methylated genes and those with unmethylated genes, with two notable exceptions: it was significantly shorter when TAC1 was methylated $(P=0.035$; Fig. $4 \mathrm{~b})$ and when $G A L$ was methylated $(P=0.021$; Fig. $4 \mathrm{e})$. Based on log-rank tests, we detected an association between poor survival and the methylation phenotype defined as $\geq 3$ methylated genes $(P=0.007$; Additional file 4 : Table S2). The DFS in patients with 3-5 methylated genes was lower than that in the group with 0-5 methylated genes (28.1 versus 61 . $3 \%$, respectively; log-rank test, $P=0.007$; Fig. 4f). Sitespecific DFS time did not differ significantly in patients with methylated versus unmethylated genes, with two notable exceptions: it was significantly shorter when SST was unmethylated in laryngeal cancer (log-rank test, $P=$ 0.001) and when TAC1 was methylated in oral cavity cancer (log-rank test, $P=0.001$ ) (Additional file 5: Figure S3, Additional file 6: Figure S4, Additional file 7: Figure S5, and Additional file 8: Figure S6).

\section{Prognostic value of the methylation status of neuropeptide gene promoters}

The association between methylation and risk of recurrence was estimated via multivariate analysis by using a Cox proportional hazards model adjusted for age, sex, smoking status, alcohol consumption, and clinical stage. In patients whose TAC1 promoter was methylated (146/ $230,63.5 \%)$, the adjusted odds ratio (OR) for recurrence 
was 1.620 (95\% confidence interval [CI] 1.018-2.578, $P=0.042)$. GAL methylation $(46 / 230,20.0 \%)$ showed a significant association with the $\mathrm{OR}$ for recurrence $(\mathrm{OR}=1.692$, 95\% CI 1.063-2.694, $P=0.027$; Table 2). Dense methylation of MI (3-5) had a significantly higher OR than low methylation of MI (0-2) for recurrence of 1.756 (95\% CI 1.160-2.659, $P=0.008$; Table 2).

ORs for recurrence according to tumor origin were also determined for four sites in this study: the hypopharynx, larynx, oropharynx, and oral cavity. When the SST promoter was methylated in patients with laryngeal cancers, the OR was 0.080 (95\% CI $0.018-0.349 ; P=0$. 001). TAC1 methylation in oral cancer was significantly associated with the OR for recurrence (OR $=4.427,95 \%$ CI 1.634-12.00, $P=0.003)$. In patients who had laryngeal cancer and whose $H C R T$ promoter was methylated, the adjusted OR for recurrence was 3.280 (95\% CI 1.054-10. $21 ; P=0.040)$. Methylation of the $G A L$ promoters was positively correlated with recurrence in patients with oropharyngeal cancers $(\mathrm{OR}=3.006,95 \% \mathrm{CI} 1.134-7.968$, $P=0.027$; Fig. 5).

\section{External validation of results by using data from the TCGA database}

The methylation status of the five neuropeptide gene promoters was determined in an additional 516 HNSCC samples and 50 normal samples (Additional file 9: Figure S7). The average $\beta$ values for SST, TAC1, NPY, and GAL methylation were significantly higher in the HNSCC samples than in the normal samples $(P<0.05)$. Methylation of the HCRT and GAL promoters was not associated with

Table 2 Methylation status of individual genes and associations with disease-free survival using Cox proportional hazards model in 230 patients

\begin{tabular}{|c|c|c|c|c|c|}
\hline \multirow[t]{2}{*}{ Gene } & \multirow{2}{*}{$\begin{array}{l}\text { Methylation } \\
\text { status }\end{array}$} & \multirow[t]{2}{*}{ Overall(\%) } & \multicolumn{2}{|c|}{ Recurrence events } & \multirow{2}{*}{$\begin{array}{l}\text { Adjusted RR } \\
\left(95 \% \mathrm{Cl}^{\dagger}{ }^{\dagger}\right.\end{array}$} \\
\hline & & & $\begin{array}{l}\text { Positive } \\
(N=91)\end{array}$ & $\begin{array}{l}\text { Negative } \\
(N=139)\end{array}$ & \\
\hline \multirow[t]{2}{*}{ SST } & Yes & 194(84.3) & 78 & 116 & \\
\hline & No & $36(15.7)$ & 13 & 23 & $0.847(0.470-1.526)$ \\
\hline \multirow[t]{2}{*}{ TAC1 } & Yes & $146(63.5)$ & 65 & 81 & \\
\hline & No & $84(36.5)$ & 26 & 58 & $1.620(1.018-2.578)^{*}$ \\
\hline \multirow[t]{2}{*}{ HCRT } & Yes & $75(32.6)$ & 37 & 38 & \\
\hline & No & $155(67.4)$ & 54 & 101 & $1.235(0.805-1.895)$ \\
\hline \multirow[t]{2}{*}{ NPY } & Yes & $65(28.3)$ & 26 & 39 & \\
\hline & No & $165(71.7)$ & 65 & 100 & $1.126(0.713-1.779)$ \\
\hline \multirow[t]{2}{*}{ GAL } & Yes & $46(20.0)$ & 27 & 19 & \\
\hline & No & 184(80.0) & 64 & 120 & $1.692(1.063-2.694)^{*}$ \\
\hline \multirow[t]{2}{*}{ Ml } & $3-5$ & $104(45.2)$ & 53 & 51 & \\
\hline & $0-2$ & $126(54.8)$ & 38 & 88 & $1.756(1.160-2.659)^{*}$ \\
\hline
\end{tabular}

* $P<0.05$

${ }^{\dagger}$ Adjusted for age, gender, smoking status, alcohol exposure, and stage patients with HNSCC or the normal control group. A Pearson correlation revealed significant correlations between mRNA expression and DNA methylation of SST, $T A C 1$, and NPY, but not correlations of HCRT and GAL (Additional file 10: Figure S8). Recurrence events were significantly associated with $H C R T$ methylation $(P=0.005)$ and $G A L$ methylation $(P=0.031)$ in laryngeal cancer patients (Additional file 11: Table S3).

\section{Discussion}

Identifying epigenetic modifications in SST, TAC1, $H C R T$, NPY, and GAL is important for understanding how tumors arise and whether they will recur. Using real-time PCR, we examined the methylation status of these genes, all of which encode neuropeptide genes, in 230 HNSCCs originating in the hypopharynx, larynx, oropharynx, or oral cavity. We found that aberrant methylation of the TAC1 and GAL promoters was positively correlated with recurrence in patients with HNSCCs. To our knowledge, this study is the first to analyze whether HNSCC primary tumors originating from different anatomic sites exhibit similar DNA methylation changes or whether DNA methylation events are specific to the anatomic site.

SST and the somatostatin receptors have been identified as tumor suppressor genes that possess potent antitumor and antisecretory activities in several human cancers [15]. Hypermethylation of SST has been described in colon cancer [16], gastric cancer [17], esophageal cancer [18], and head and neck cancer [9]. TAC1 encodes the neuropeptides substance $\mathrm{P}$, neurokinin $\mathrm{A}$, and neurokinin $B$, which act through three types of tachykinin receptors (TACR1, TACR2, and TACR3) [19]. Hypermethylation of TAC1 is associated with poor prognosis in esophageal cancer, breast cancer, and colorectal cancer [20-22]. HCRT encodes a hypothalamic neuropeptide precursor protein that gives rise to two mature neuropeptides, orexin A and orexin B [23]. Orexins induce high levels of apoptosis, resulting in a massive reduction in cell growth in various cancer cell lines acting at HCRTR1 or HCRTR2 [24]. NPY activates five GPCRs, namely, NPY1R, NPY2R, NPY4R, NPY5R, and NPY6R [25]. It is one of the most abundantly distributed neurotransmitters and vasoconstrictors in the central and peripheral nervous systems. Hypermethylation of $N P Y$ has been described in several cancers, including renal cell carcinoma [26], breast cancer [27], and colorectal cancer [28]. GAL is involved in the regulation of many physiological functions, such as feeding, metabolism, and body weight control [29]. DNA methylation of GAL in the prefrontal cortex plays a role in major depressive disorder [30]. In HNSCC, recent data are conflicting, with significant GAL overexpression reported in tumor samples [31], whereas our previous study proposed that 


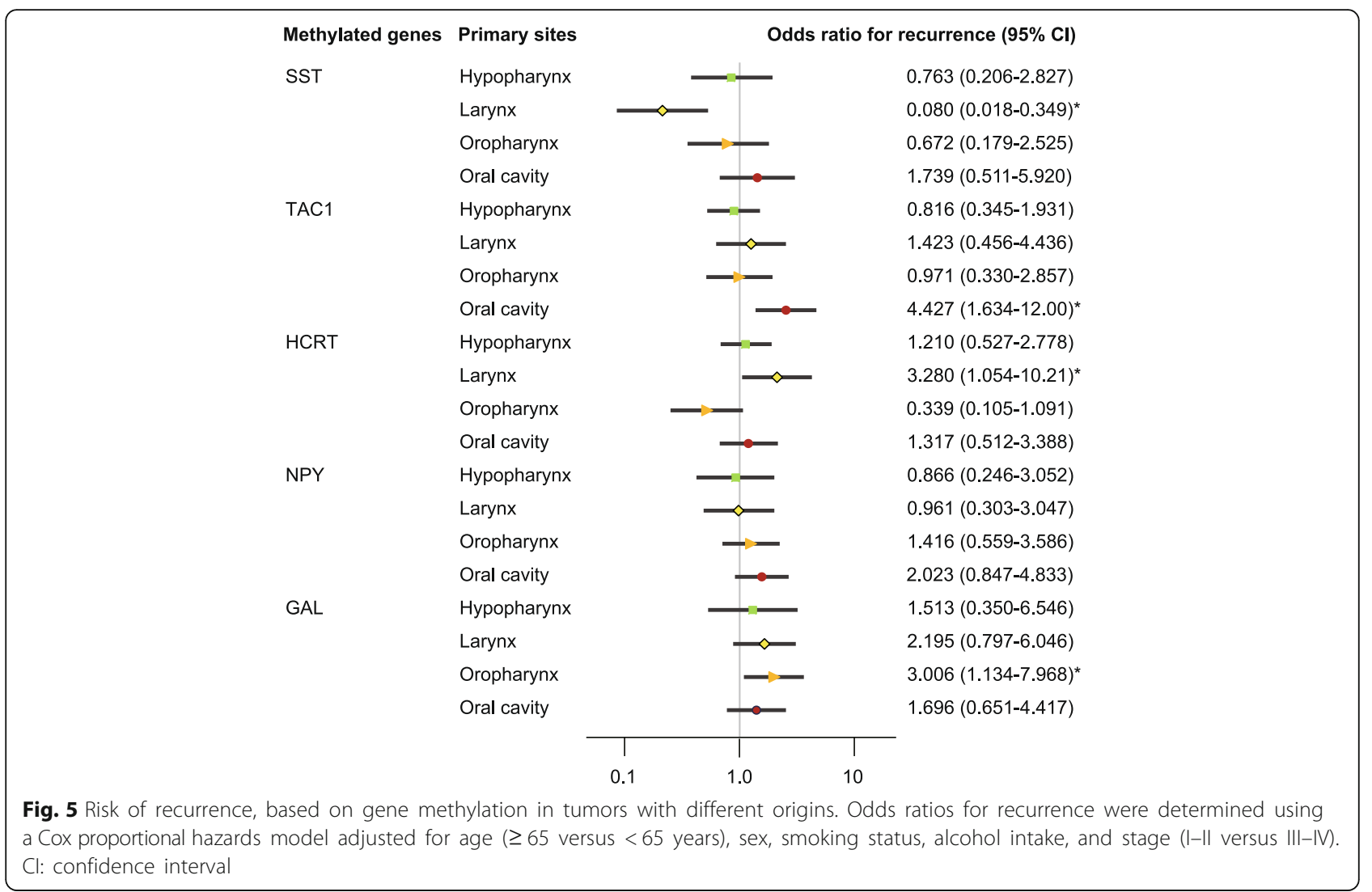

GAL promoter methylation and gene silencing is correlated with significantly lower DFS and growth suppression of HNSCC cells after forced gene expression [32].

Neuropeptides have become a focus area of research because of the distinct GPCR activation, potent and varied biological activities, and potential therapeutic targets [33]. A novel approach to treat insomnia, the most common sleep disorder, has been introduced recently with the approval of suvorexant, the first in a new class of orexin receptor (HCRTR) antagonists [34]. Newly developed neurokinin-1 receptor antagonists, especially aprepitant, have been recently analyzed for the prevention of postoperative nausea and vomiting [35]. Certain neuropeptides, which are natural compounds by origin, possess powerful antitumorigenic and tumor suppressor properties, thereby providing added benefits for future potential therapeutic strategies [36]. Hence, innovating to obtain more potent selective ligands (agonists) will be a focus area for specific tumor targeting in the future.

HNSCC is the most complex "organ site," affecting different anatomic sites of the upper aerodigestive tract such as the oral cavity, larynx, and naso-, oro-, and hypopharynx [37]. Patients with HNSCC often present a long history of tobacco and alcohol use.
Recently, human papillomavirus (HPV) infection has emerged as an additional risk factor [38]. Many other risk factors are also related to HNSCC; therefore, it is necessary to investigate the profiles for different anatomic sites [39, 40].

\section{Conclusion}

The current study provides evidence that the methylation status of SST, TAC1, HCRT, NPY, and GAL is an independent prognostic factor for DFS in patients with HNSCC. Further analyses showed that the aberrant methylation of SST may be a potential marker for patients with laryngeal cancer that is at low risk of relapse. Moreover, to our knowledge, our study is the first to suggest that TAC1, HCRT, and GAL methylation is associated with worse DFS and that this may be a critical event in oral cancers, laryngeal cancers, and oropharyngeal cancers, respectively. This study involving human specimens and high-throughput profiling platforms may be susceptible to measurement bias from various sources. Our findings support the use of methylation markers in patient selection for adjuvant therapy after initial surgical treatment; however, our preliminary findings need to be validated in larger and more homogeneous HNSCC patient cohorts. 


\section{Additional files}

Additional file 1: Table S1. Real-time MSP primer list. (DOCX $20 \mathrm{~kb}$ )

Additional file 2: Figure S1. Schematic representation of methylation analysis of 0 on 5 genes by qMSP. Schematic representation of (a) SST, (b) TAC1, (c) HCRT, (d) NPY, and (e) GAL genes. Exon structures and CpG sites within expanded views of the promoter region relative to the transcription start site (TSS). Vertical lines, individual CpG sites; straight arrows, relative location of the primers used for qMSP; bent arrow, TSS; arrowhead, translation start site (ATG). (EPS $1793 \mathrm{~kb}$ )

Additional file 3: Figure S2. ROC curves for the methylation markers in head and neck carcinomas versus adjacent normal mucosal tissue. On the basis of the ROC curve analysis, the sensitivity, specificity, and cutoff level were determined to be $80.6 \%, 94.4 \%$, and 0.046 for SST (a): $72.2 \%$, 97.2\%, and 0.08 for TAC1 (b); 67.6\%, 97.2\%, and 0.099 for HCRT (c); $50.0 \%$, 97.2\%, and 0.041 for NPY (d); and 25.0\%, 86.1\%, and 0.100 for GAL (e). (EPS $1057 \mathrm{~kb}$ )

Additional file 4: Table S2. Results of log-rank tests for the effect of number of methylated genes on disease-free survival in 230 HNSCC cases. (DOCX 14 kb)

Additional file 5: Figure S3. Kaplan-Meier survival curves for the 58 patients with hypopharyngeal cancer, according to the methylation status of the five target genes. Disease-free survival for (a) SST, (b) TAC1, (c) HCRT, (d) NPY, and (e) GAL in the case of methylated (red lines) and unmethylated (blue lines) genes. ( $f$ ) Joint analysis of the 5 genes. Blue line: patients with 0-2 methylated genes; red line: patients with 3-5 methylated genes. A probability of $<0.05\left({ }^{*} P<0.05\right)$ was considered to represent a statistically significant difference. (EPS $1460 \mathrm{~kb}$ )

Additional file 6: Figure S4. Kaplan-Meier survival curves for the 45 patients with laryngeal cancer, according to the methylation status of the five target genes. Disease-free survival for (a) SST, (b) TAC1, (c) HCRT, (d) $N P Y$, and (e) GAL in the case of methylated (red lines) and unmethylated (blue lines) genes. (f) Joint analysis of the 5 genes. Blue line: patients with 0-2 methylated genes; red line: patients with 3-5 methylated genes. A probability of $<0.05\left({ }^{*} P<0.05\right)$ was considered to represent a statistically significant difference. (EPS $1471 \mathrm{~kb}$ )

Additional file 7: Figure S5. Kaplan-Meier survival curves for the 56 patients with oropharyngeal cancer, according to the methylation status of the five target genes. Disease-free survival for (a) SST, (b) TAC1, (c) HCRT, (d) NPY, and (e) GAL in the case of methylated (red lines) and unmethylated (blue lines) genes. (f) Joint analysis of the 5 genes. Blue line: patients with 0-2 methylated genes; red line: patients with 3-5 methylated genes. A probability of $<0.05\left({ }^{*} P<0.05\right)$ was considered to represent a statistically significant difference. (EPS $1460 \mathrm{~kb}$ )

Additional file 8: Figure S6. Kaplan-Meier survival curves for the 71 patients with oral cavity cancer, according to the methylation status of the five target genes. Disease-free survival for (a) SST, (b) TAC1, (c) HCRT, (d) NPY, and (e) GAL in the case of methylated (red lines) and unmethylated (blue lines) genes. (f) Joint analysis of the 5 genes. Blue line: patients with 0-2 methylated genes; red line: patients with 3-5 methylated genes. A probability of $<0.05\left({ }^{*} P<0.05\right)$ was considered to represent a statistically significant difference. (EPS $1465 \mathrm{~kb}$ )

Additional file 9: Figure S7. DNA methylation data from The Cancer Genome Atlas database. DNA methylation data for SST, TAC1, HCRT, NPY, and GAL were collected from the TCGA database of DNA methylation and gene expression in human cancer (http://methhc.mbc.nctu.edu.tw/ php/index.php). ${ }^{*} P<0.05$. (EPS $896 \mathrm{~kb}$ )

Additional file 10: Figure S8. Methylation and expression status of the five neuropeptide genes in HNSCCs in the TCGA database. Scatter plot analysis for (A) SST, (B) TAC1, (C) HCRT, (D) NPY, and (E) GAL. Spearman rank correlation coefficient (R2) and $P$ values are shown. (EPS $2568 \mathrm{~kb}$ )

Additional file 11: Table S3. Distribution of methylation status by recurrence events in TCGA cohort. (DOCX $17 \mathrm{~kb}$ )

\section{Abbreviations}

ACTB: $\beta$-Actin; Cl: Confidence interval; DFS: Disease-free survival; GAL: Galanin; GPCR: G protein-coupled receptors; HCRT: Hypocretin neuropeptide precursor; HNSCC: Head and neck squamous cell cancer;
HPV: Human papilloma virus; MI: Methylation index; NMV: Normalized methylation value; NPY: Neuropeptide Y; OR: Odds ratios; Q-MSP: Quantitative methylation-specific PCR; ROC: Receiver operating characteristic; SST: Somatostatin; TAC1: Tachykinin precursor 1; TACR: Tachykinin receptor; TCGA: The Cancer Genome Atlas

\section{Acknowledgements}

We thank Ms. Yuko Mohri for the technical assistance.

\section{Funding}

This work was supported by Grants-in-Aid for Scientific Research (nos. 16K11228, 16K20239, 17K11380, 17K16903, and 17K16904) from the Ministry of Education, Culture, Sports, Science, and Technology of Japan.

\section{Availability of data and materials}

The datasets obtained and/or analyzed during the current study are available from the corresponding author upon a reasonable request.

\section{Authors' contributions}

KM conceptualized the study and contributed to the methodology of the study. MM, YM, and RI contribute to the software used for the study. Al validated the study. DM participated in the formal analysis of the study. TK and SE participated in the investigation of the study. $\mathrm{HM}$ and $\mathrm{MM}$ contributed to the resources used for the study. KM wrote the original draft of the manuscript. TK wrote, reviewed, and edited the manuscript. KM participated in the visualization of the study. HM supervised the study. KM was responsible for the project administration and carried out the funding acquisition of the study. All authors read and approved the final manuscript.

\section{Ethics approval and consent to participate}

These tissue samples were obtained with informed written consent following protocols approved by the research ethics board of the Hamamatsu University School of Medicine.

\section{Consent for publication}

Consent for publication was obtained from all patients.

\section{Competing interests}

The authors declare that they have no competing interests.

\section{Publisher's Note}

Springer Nature remains neutral with regard to jurisdictional claims in published maps and institutional affiliations.

\section{Author details}

${ }^{1}$ Department of Otolaryngology/Head and Neck Surgery, Hamamatsu University School of Medicine, 1-20-1 Handayama, Hamamatsu, Shizuoka 431-3192, Japan. ²Department of Otolaryngology/Head and Neck Surgery, Jichi Medical University, Tochigi, Japan.

Received: 29 December 2017 Accepted: 28 March 2018 Published online: 11 April 2018

\section{References}

1. Heng BC, Aubel D, Fussenegger M. An overview of the diverse roles of G-protein coupled receptors (GPCRs) in the pathophysiology of various human diseases. Biotechnol Adv. 2013;31(8):1676-94.

2. Xu TR, Yang Y, Ward R, Gao L, Liu Y. Orexin receptors: multi-functional therapeutic targets for sleeping disorders, eating disorders, drug addiction, cancers and other physiological disorders. Cell Signal. 2013;25(12):2413-23.

3. Armaiz-Pena GN, Lutgendorf SK, Cole SW, Sood AK. Neuroendocrine modulation of cancer progression. Brain Behav Immun. 2009;23(1):10-5.

4. Galoian K, Patel P. Epigenetic control of cancer by neuropeptides. Biomed Rep. 2017;6(1):3-7

5. Lappano R, Maggiolini M. G protein-coupled receptors: novel targets for drug discovery in cancer. Nat Rev Drug Discov. 2011;10(1):47-60.

6. Liu Y, An S, Ward R, Yang Y, Guo XX, Li W, Xu TR. G protein-coupled receptors as promising cancer targets. Cancer Lett. 2016;376(2):226-39.

7. Munoz M, Covenas R. Neurokinin-1 receptor: a new promising target in the treatment of cancer. Discov Med. 2010;10(53):305-13. 
8. Misawa Y, Misawa K, Kawasaki H, Imai A, Mochizuki D, Ishikawa R, Endo S, Mima M, Kanazawa T, Iwashita T, et al. Evaluation of epigenetic inactivation of vascular endothelial growth factor receptors in head and neck squamous cell carcinoma. Tumour Biol. 2017;39(7):1010428317711657.

9. Misawa K, Misawa $Y$, Kondo H, Mochizuki D, Imai A, Fukushima H, Uehara T, Kanazawa T, Mineta $\mathrm{H}$. Aberrant methylation inactivates somatostatin and somatostatin receptor type 1 in head and neck squamous cell carcinoma. PLoS One. 2015;10(3):e0118588.

10. Misawa K, Kanazawa T, Misawa Y, Imai A, Uehara T, Mochizuki D, Endo S, Takahashi G, Mineta H. Frequent promoter hypermethylation of tachykinin-1 and tachykinin receptor type 1 is a potential biomarker for head and neck cancer. J Cancer Res Clin Oncol. 2013;139(5):879-89.

11. Misawa K, Mochizuki D, Endo S, Mima M, Misawa Y, Imai A, Shinmura K, Kanazawa T, Carey TE, Mineta H. Site-specific methylation patterns of the GAL and GALR1/2 genes in head and neck cancer: potential utility as biomarkers for prognosis. Mol Carcinog. 2017;56(3):1107-16.

12. Misawa K, Mochizuki D, Imai A, Endo S, Mima M, Misawa Y, Kanazawa T, Carey TE, Mineta $\mathrm{H}$. Prognostic value of aberrant promoter hypermethylation of tumor-related genes in early-stage head and neck cancer. Oncotarget. 2016;7(18):26087-98.

13. Misawa K, Imai A, Mochizuki D, Misawa Y, Endo S, Hosokawa S, Ishikawa R, Mima M, Shinmura K, Kanazawa T, et al. Genes encoding neuropeptide receptors are epigenetic markers in patients with head and neck cancer: a site-specific analysis. Oncotarget. 2017;8(44):76318-28.

14. Huang WY, Hsu SD, Huang HY, Sun YM, Chou CH, Weng SL, Huang HD. MethHC: a database of DNA methylation and gene expression in human cancer. Nucleic Acids Res. 2015;43(Database issue):D856-61.

15. Reubi JC, Laissue JA. Multiple actions of somatostatin in neoplastic disease. Trends Pharmacol Sci. 1995;16(3):110-5.

16. Mori $Y$, Cai $K$, Cheng Y, Wang S, Paun B, Hamilton JP, Jin Z, Sato F, Berki AT, Kan $T$, et al. A genome-wide search identifies epigenetic silencing of somatostatin, tachykinin-1, and 5 other genes in colon cancer. Gastroenterology. 2006;131(3):797-808.

17. Jackson K, Soutto M, Peng D, Hu T, Marshal D, El-Rifai W. Epigenetic silencing of somatostatin in gastric cancer. Dig Dis Sci. 2011;56(1):125-30.

18. Jin Z, Mori Y, Hamilton JP, Olaru A, Sato F, Yang J, Ito T, Kan T, Agarwal R, Meltzer SJ. Hypermethylation of the somatostatin promoter is a common, early event in human esophageal carcinogenesis. Cancer. 2008;112(1):43-9.

19. Pennefather JN, Lecci A, Candenas ML, Patak E, Pinto FM, Maggi CA. Tachykinins and tachykinin receptors: a growing family. Life Sci. 2004;74(12):1445-63.

20. Jin Z, Olaru A, Yang J, Sato F, Cheng Y, Kan T, Mori Y, Mantzur C, Paun B, Hamilton JP, et al. Hypermethylation of tachykinin-1 is a potential biomarker in human esophageal cancer. Clin Cancer Res. 2007;13(21):6293-300.

21. Jeschke J, Van Neste L, Glockner SC, Dhir M, Calmon MF, Deregowski V, Van Criekinge W, Vlassenbroeck I, Koch A, Chan TA, et al. Biomarkers for detection and prognosis of breast cancer identified by a functional hypermethylome screen. Epigenetics. 2012;7(7):701-9.

22. Tham C, Chew M, Soong R, Lim J, Ang M, Tang C, Zhao Y, Ong SY, Liu Y. Postoperative serum methylation levels of TAC1 and SEPT9 are independent predictors of recurrence and survival of patients with colorectal cancer. Cancer. 2014:120(20):3131-41.

23. Sakurai T, Moriguchi T, Furuya K, Kajiwara N, Nakamura T, Yanagisawa M, Goto K. Structure and function of human prepro-orexin gene. J Biol Chem. 1999;274(25):17771-6.

24. Rouet-Benzineb P, Rouyer-Fessard C, Jarry A, Avondo V, Pouzet C, Yanagisawa M, Laboisse C, Laburthe M, Voisin T. Orexins acting at native OX(1) receptor in colon cancer and neuroblastoma cells or at recombinant OX(1) receptor suppress cell growth by inducing apoptosis. J Biol Chem. 2004;279(44): 45875-86.

25. Ejaz A, LoGerfo FW, Khabbaz K, Pradhan L. Expression of neuropeptide $Y$ substance $P$, and their receptors in the right atrium of diabetic patients. Clin Transl Sci. 2011;4(5):346-50.

26. Mendoza-Perez J, Gu J, Herrera LA, Tannir NM, Zhang S, Matin S, Karam JA Wood CG, Wu X. Prognostic significance of promoter CpG island methylation of obesity-related genes in patients with nonmetastatic renal cell carcinoma. Cancer. 2017;123(18):3617-27.

27. Li Z, Heng J, Yan J, Guo X, Tang L, Chen M, Peng L, Wu Y, Wang S, Xiao Z, et al. Integrated analysis of gene expression and methylation profiles of 48 candidate genes in breast cancer patients. Breast Cancer Res Treat. 2016;160(2):371-83.
28. Roperch JP, Benzekri K, Mansour $\mathrm{H}$, Incitti R. Improved amplification efficiency on stool samples by addition of spermidine and its use for non-invasive detection of colorectal cancer. BMC Biotechnol. 2015;15:41.

29. Gundlach AL. Galanin/GALP and galanin receptors: role in central control of feeding, body weight/obesity and reproduction? Eur J Pharmacol. 2002; 440(2-3):255-68.

30. Barde S, Ruegg J, Prud'homme J, Ekstrom TJ, Palkovits M, Turecki G, Bagdy G, Ihnatko R, Theodorsson E, Juhasz G, et al. Alterations in the neuropeptide galanin system in major depressive disorder involve levels of transcripts, methylation, and peptide. Proc Natl Acad Sci U S A. 2016;113(52):E8472-81.

31. Sugimoto T, Seki N, Shimizu S, Kikkawa N, Tsukada J, Shimada H, Sasaki K, Hanazawa T, Okamoto Y, Hata A. The galanin signaling cascade is a candidate pathway regulating oncogenesis in human squamous cell carcinoma. Genes Chromosomes Cancer. 2009;48(2):132-42.

32. Misawa K, Kanazawa T, Misawa Y, Uehara T, Imai A, Takahashi G, Takebayashi S, Cole A, Carey TE, Mineta H. Galanin has tumor suppressor activity and is frequently inactivated by aberrant promoter methylation in head and neck cancer. Transl Oncol. 2013;6(3):338-46.

33. Brain SD, Cox HM. Neuropeptides and their receptors: innovative science providing novel therapeutic targets. Br J Pharmacol. 2006;147(Suppl 1):S202-11.

34. Dubey AK, Handu SS, Mediratta PK. Suvorexant: the first orexin receptor antagonist to treat insomnia. J Pharmacol Pharmacother. 2015;6(2):118-21.

35. Liu M, Zhang H, Du BX, Xu FY, Zou Z, Sui B, Shi XY. Neurokinin-1 receptor antagonists in preventing postoperative nausea and vomiting: a systematic review and meta-analysis. Medicine (Baltimore). 2015;94(19):e762.

36. Munoz M, Covenas R. Targeting NK-1 receptors to prevent and treat pancreatic cancer: a new therapeutic approach. Cancers (Basel). 2015;7(3):1215-32.

37. Echarri MJ, Lopez-Martin A, Hitt R. Targeted therapy in locally advanced and recurrent/metastatic head and neck squamous cell carcinoma (LA-R/M HNSCC). Cancers. 2016:8(3):E27.

38. Dok R, Nuyts S. HPV positive head and neck cancers: molecular pathogenesis and evolving treatment strategies. Cancers. 2016;8(4):E41.

39. Belbin TJ, Schlecht NF, Smith RV, Adrien LR, Kawachi N, Brandwein-Gensler M Bergman A, Chen Q, Childs G, Prystowsky MB. Site-specific molecular signatures predict aggressive disease in HNSCC. Head Neck Pathol. 2008;2(4):243-56.

40. Galbiatti AL, Padovani-Junior JA, Maniglia JV, Rodrigues CD, Pavarino EC, Goloni-Bertollo EM. Head and neck cancer: causes, prevention and treatment. Braz J Otorhinolaryngol. 2013;79(2):239-47.

\section{Submit your next manuscript to BioMed Central and we will help you at every step:}

- We accept pre-submission inquiries

- Our selector tool helps you to find the most relevant journal

- We provide round the clock customer support

- Convenient online submission

- Thorough peer review

- Inclusion in PubMed and all major indexing services

- Maximum visibility for your research

Submit your manuscript at www.biomedcentral.com/submit 